\title{
Frequency and clinical features of treatment-refractory myasthenia gravis
}

\author{
Jakob Rath ${ }^{1}$ (1) - Ines Brunner ${ }^{1}$ Matthias Tomschik ${ }^{1}$. Gudrun Zulehner ${ }^{1}$. Eva Hilger ${ }^{1}$. Martin Krenn ${ }^{1}$. Anna Paul ${ }^{1}$. \\ Hakan Cetin ${ }^{1} \cdot$ Fritz Zimprich $^{1}$
}

Received: 6 November 2019 / Accepted: 4 December 2019 / Published online: 11 December 2019

(c) The Author(s) 2019

\begin{abstract}
Background To investigate the frequency and characterize the clinical features of treatment-refractory myasthenia gravis in an Austrian cohort.

Methods Patient charts of 126 patients with generalized myasthenia gravis and onset between 2000 and 2016 were analyzed retrospectively. Patients were classified as treatment-refractory according to strict, predefined criteria. These mandated patients being at least moderately symptomatic (i.e., MGFA class III) or needing either maintenance immunoglobulins or plasma exchange therapy for at least 1 year in spite of two adequately dosed immunosuppressive drugs. Clinical features and outcome at last follow-up were compared to treatment-responsive patients.

Results 14 out of 126 patients $(11.1 \%)$ met these criteria of treatment-refractory myasthenia gravis. Treatment-refractory patients had more frequent clinical exacerbations and more often received rescue treatments or a further escalation of immunosuppressive therapies. They also remained more severely affected at last follow-up. An early onset of myasthenia gravis was associated with a higher risk for a refractory course.

Conclusion A small subgroup of patients with generalized myasthenia gravis do not respond sufficiently to standard therapies. Refractory disease has considerable implications for both patients and health care providers and highlights an unmet need for new treatment options.
\end{abstract}

Keywords Myasthenia gravis $\cdot$ Outcome $\cdot$ Refractory disease $\cdot$ Treatment

$\begin{array}{ll}\text { Abbreviations } \\ \text { AChR } & \text { Acetylcholine receptor } \\ \text { CI } & \text { Confidence interval } \\ \text { EOMG } & \text { Early onset myasthenia gravis } \\ \text { IA } & \text { Immunoadsorption } \\ \text { IQR } & \text { Interquartile range } \\ \text { LOMG } & \text { Late onset myasthenia gravis } \\ \text { MG } & \text { Myasthenia gravis } \\ \text { MGFA } & \text { Myasthenia Gravis Foundation of America } \\ \text { MuSK } & \text { Muscle-specific tyrosine kinase } \\ \text { OR } & \text { Odds ratio } \\ \text { PLEX } & \text { Plasma exchange therapy }\end{array}$

Fritz Zimprich

friedrich.zimprich@meduniwien.ac.at

1 Department of Neurology, Medical University of Vienna, Währinger Gürtel 18-20, 1090 Vienna, Austria

\section{Introduction}

Myasthenia gravis is an autoimmune disease of the neuromuscular junction with a prevalence of around 16 per 100,000 [1]. Patients are grouped according to the age at onset, presence of a specific antibody, thymus pathology, and distribution of symptoms [2]. The majority of patients (approximately 80\%) have antibodies against the nicotinic acetylcholine receptor (AChR), while in a small subset of patients antibodies against muscle-specific receptor tyrosine kinase (MuSK), lipoprotein-related protein 4 (LRP4) or other postsynaptic structures of the neuromuscular junction are detected. In about $5 \%$ of patients, no antibodies are found. Additionally, paraneoplastic disease can occur in patients with thymoma that leads to generalized thymomaassociated myasthenia gravis with the detection of AChRantibodies in nearly all patients $[3,4]$.

The natural, untreated course of myasthenia gravis has been associated with a high mortality and a persistence of symptoms in most patients $[5,6]$, but the introduction of 
immunosuppressive treatments, thymectomy in selected patients, modern intensive care medicine as well as the availability of rescue treatments such as intravenous immunoglobulins (IVIG), plasma exchange therapy (PLEX) or immunoadsorption (IA) has greatly improved the outcome across all subgroups of patients [7]. However, approximately $10-15 \%$ of patients still show a poor response to available standard treatments and consequently continue to suffer from disabling symptoms. They also experience frequent disease exacerbations leading to a reduced quality of life and frequent admissions to hospitals and emergency departments [8-11]. In addition, the necessary treatment with high-dose immunosuppressive drugs is often associated with side effects, which negatively affects patients' quality of life.

So far, only a few studies have specifically addressed the characteristics of treatment-resistance myasthenia gravis patients [12-14]. It is also an open question which factors predispose patients to a refractory disease course with some observations suggesting an early onset, female gender, an association with thymoma or the presence of MuSK-antibodies as risk factors $[13,14]$. Given the unmet clinical needs in treatment-refractory patients, a further characterization and definition of this subgroup is clearly warranted to recognize and select patients early for a targeted management with modern immunosuppressive drugs $[15,16]$.

The aim of this retrospective study was to evaluate the frequency of treatment-refractory disease courses among patients with generalized myasthenia gravis according to a strict definition and assess the clinical features of these patients.

\section{Methods}

\section{Patients}

We retrospectively investigated charts of patients with onset of myasthenia gravis between 2000 and 2016, who were treated at our tertiary neuromuscular center at the Department of Neurology of the Medical University of Vienna. We included only patients with sufficient follow-up data of at least 2 years and generalized myasthenia gravis within the first year after onset. Diagnostic criteria for myasthenia gravis consisted of typical myasthenic symptoms in combination with myasthenia gravis-related antibodies, or in seronegative patients either pathological repetitive nerve stimulation with a decrement over $10 \%$, a positive edrophonium chloride test, or documented clinical improvement following pyridostigmine treatment. Disease severity was retrospectively assessed at documented time points using the criteria by the Myasthenia Gravis Foundation of America (MGFA) class [17]. Ethical approval was obtained from the Ethics Committee of the Medical University of Vienna.

\section{Outcome measures}

The primary outcome measure was the occurrence of treatment-refractory MG.

We defined treatment-refractory myasthenia gravis at the earliest 2 years after diagnosis as soon as the following conditions were met:

1. Persistent moderate to severe myasthenic symptoms (i.e., $\geq$ MGFA class III) for the last 12 months $O R$

2. MGFA class $<$ III but requirement of regular maintenance treatment with IVIG or PLEX/IA for the last 12 months in combination with

Treatment with at least 2 concurrent long-term immunosuppressive drugs at adequate doses for the last 12 months.

Long-term immunosuppressive drugs included all conventional therapies including corticosteroids, azathioprine, mycophenolate-mofetil, and tacrolimus. The average prednisone-equivalent dose had to be at least $\geq 5 \mathrm{mg}$ daily. Escalation treatment with rituximab and pulsed cyclophosphamide given according to standard regimens was regarded as equivalent to the treatment with 1 conventional immunosuppressive drug for 12 months.

Secondary outcome measures were MGFA class, MGFA postintervention status for asymptomatic patients, treatment at last follow-up and all-cause mortality. Additionally, the number of myasthenic crises (MGFA class $\mathrm{V})$ and severe exacerbations of myasthenic symptoms (defined as clinical deterioration requiring acute medical intervention or inpatient treatment but without the need for mechanical ventilation) was assessed. Furthermore, the number of rescue treatments with IVIG or PLEX/IA during the course of disease and occurrence of severe side effects of immunosuppressive treatments was analyzed.

\section{Statistical analysis}

Statistical analysis was performed with SPSS 24 software package (IBM, Corp. Released 2016. IBM SPSS Statistics for Macintosh, Version 24.0. Armonk, NY: IBM Corp.). Baseline variables as well as outcome measures of treatment-refractory and treatment-responsive patients were compared using the Student's $t$ test or Mann-Whitney $U$ test for continuous variables and Chi-squared test for categorical variables.

Multivariate logistic regression analyses were used to test for clinical variables associated with the occurrence of treatment-refractory myasthenia gravis. Covariates were selected according to clinical meaningful aspects. The 
following covariates and their interactions were included in the final model: EOMG vs. LOMG, sex, antibody status, and thymus histology indicating thymoma-associated myasthenia gravis. A $p$ value of $\leq 0.05$ was considered statistically significant; correction for multiple comparisons for analyses of secondary outcome measures was done using Bonferroni correction resulting in a $p$ value of $\leq 0.004$.

\section{Results}

126 patients ( 54 men, 72 women; median age at onset 49.5 , interquartile range (IQR) 37, total range (13-85) were analyzed retrospectively. Of these, $14(11.1 \%)$ patients were classified as treatment-resistant myasthenia gravis (see Fig. 1 for distribution of patients according to the subgroups proposed by Gilhus et al. [2] and Table 3 for detail description of individual patient characteristics).

9 of the 14 patients met the criteria because of persistent myasthenic symptoms and 5 patients because they required maintenance IVIG or PLEX/IA treatment. Patients met the criteria of treatment-resistant myasthenia gravis after a median of 44.5 months (IQR 40 months, total range 24-197 months). Of the 14 treatment-refractory patients, 8 were diagnosed in the first half (01 January, 2000-31 June, 2008 ) and 6 in the second half (01 July, 2008-31 December, $2016)$ of the analyzed time period $(p=0.12)$.

Baseline variables for all patients as well for the treatment response-groups are shown in Table 1. Baseline variables did not differ significantly between the two groups regarding time from onset to initiation of immunosuppressive treatment, AChR-antibody titer, MGFA class at onset,
Fig. 1 Rates of treatmentrefractory MG and treatmentresponsive $\mathrm{MG}$ according to subgroups suggested by Gilhus et al. [2]; AChR denotes acetylcholine receptor, $M G$ Myasthenia gravis and MuSK musclespecific tyrosine kinase

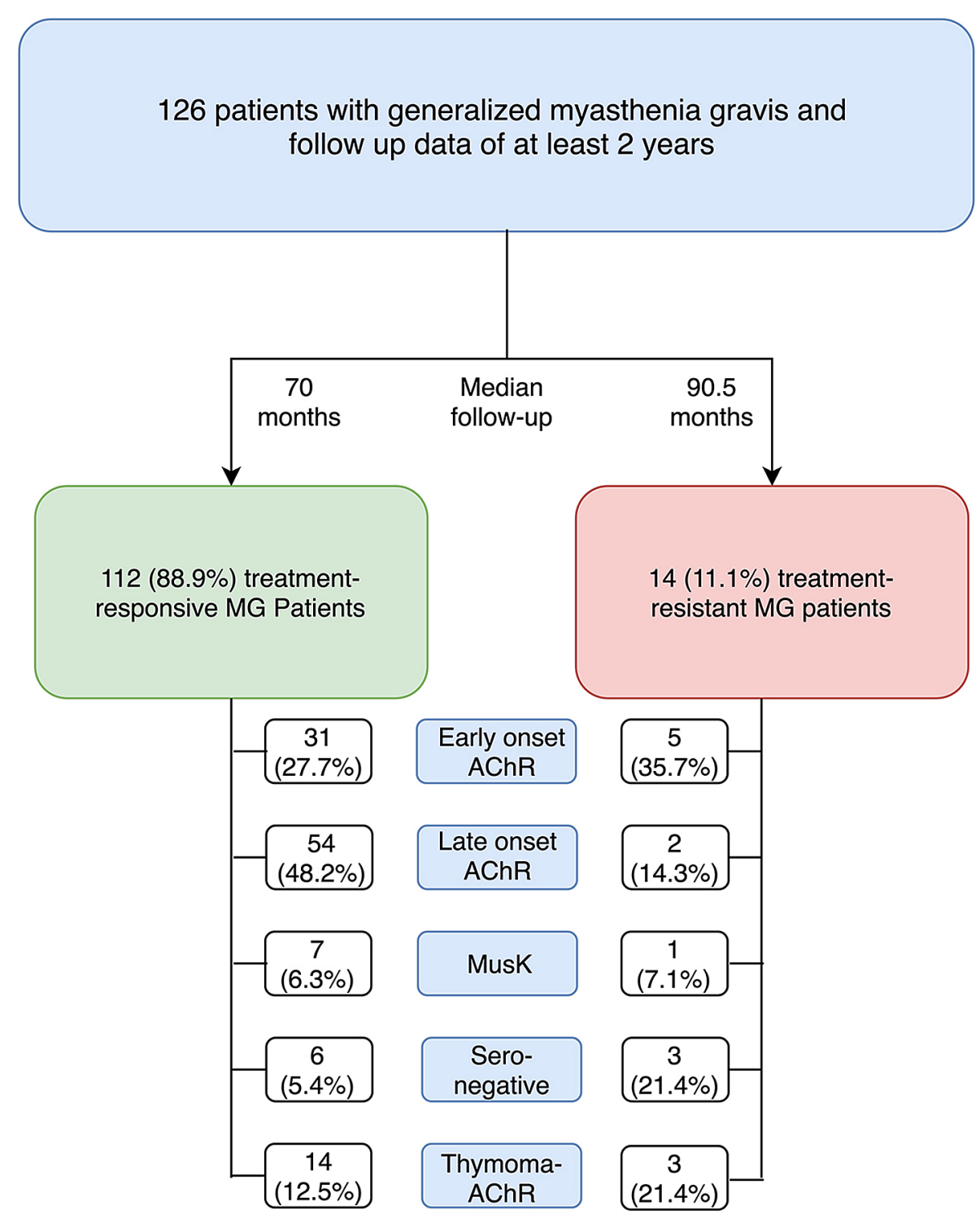


Table 1 Baseline characteristics

All patients $(n=126) \quad$ Treatment-refractory $(n=14) \quad \begin{aligned} & \text { Treatment-responsive } \quad p \text { value* } \\ & \text { (n) }\end{aligned}$
$(n=112)$

\begin{tabular}{|c|c|c|c|c|}
\hline Sex & & & & 0.25 \\
\hline Male & $54(42.9 \%)$ & $8(57.1)$ & $46(41.1 \%)$ & \\
\hline Female & $72(57.1 \%)$ & $6(42.9 \%)$ & $66(58.9 \%)$ & \\
\hline Median age & 49.5 years (IQR 37) & 33 years (IQR 25) & 50.5 (IQR 38) & $\mathbf{0 . 0 3 5}$ \\
\hline EOMG ( $<50$ years $)$ & $63(50 \%)$ & $11(78.6 \%)$ & $52(46.4 \%)$ & $\mathbf{0 . 0 2 3}^{\ddagger}$ \\
\hline Antibodies & & & & 0.085 \\
\hline $\mathrm{AChR}$ & $109(86.5 \%)$ & $10(71.4 \%)$ & $99(88.4 \%)$ & \\
\hline MuSK & $8(6.3 \%)$ & $1(7.1 \%)$ & $7(6.3 \%)$ & \\
\hline Seronegative ${ }^{* *}$ & $9(7.1 \%)$ & $3(21.4 \%)$ & $6(5.4 \%)$ & \\
\hline Median AChR-Ab titer at onset & $\begin{array}{l}17.2 \\
\text { (IQR 27.85) }\end{array}$ & 9.9 (IQR 16.6) & 17.5 (IQR 29.2) & 0.42 \\
\hline MGFA class at onset & & & & 0.96 \\
\hline 1 & $31(24.6 \%)$ & $4(28.6 \%)$ & $27(24.1 \%)$ & \\
\hline 2 & $77(61.1 \%)$ & $9(64.3 \%)$ & $68(60.7 \%)$ & \\
\hline 3 & $13(10.3 \%)$ & $1(7.1 \%)$ & $12(10.7 \%)$ & \\
\hline 4 & $3(2.4 \%)$ & 0 & $3(2.7 \%)$ & \\
\hline 5 & $2(1.6 \%)$ & 0 & $2(1.8 \%)$ & \\
\hline Max. MGFA class & & & & $<0.000^{*}$ \\
\hline 2 & $68(54 \%)$ & 0 & $68(60.7 \%)$ & \\
\hline 3 & $31(24,6 \%)$ & $9(64.3 \%)$ & $22(19.6 \%)$ & \\
\hline 4 & $18(14.3 \%)$ & $4(28.6 \%)$ & $14(12.5 . \%)$ & \\
\hline 5 & $9(7.1 \%)$ & $1(7.1 \%)$ & $8(7.1 \%)$ & \\
\hline Thymectomy & $68(54 \%)$ & $10(71.4 \%)$ & $58(51.8 \%)$ & 0.16 \\
\hline Thymus histology & & & & 0.62 \\
\hline Normal & $29(42.7 \%)$ & $3(30 \%)$ & $26(44.8 \%)$ & \\
\hline Hyperplasia & $20(29.4 \%)$ & $4(40 \%)$ & $16(27.6 \%)$ & \\
\hline Thymoma & $17(25 \%)$ & $3(30 \%)$ & $14(24.1 \%)$ & \\
\hline No data & $2(2.9 \%)$ & 0 & $2(3.5 \%)$ & \\
\hline Severe comorbidity & $23(18.3 \%)$ & $3(21.4 \%)$ & $20(17.9 \%)$ & 0.74 \\
\hline Median time onset to IST (months) & 3 (IQR 9) & 2.5 (IQR 9) & 3 (IQR 9) & 0.61 \\
\hline
\end{tabular}

Baseline characteristics of all patients and comparison of treatment-refractory and treatment-responsive patients

$A C h R$ denotes acetylcholine receptor, $M G$ myasthenia gravis, $M G F A$ Myasthenia Gravis Foundation of America, $M u S K$ muscle-specific tyrosine kinase, $E O M G$ early-onset myasthenia gravis, $I S$ immunosuppressive, $I Q R$ interquartile range, $N A$ not applicable

${ }^{*} p$ values were obtained with the Mann-Whitney $U$ or Student's $t$ test (for continuous variables) and the Chi-square test (for categorical variables) as appropriate

${ }^{* *}$ Of the 10 seronegative patients 4 [ 2 of whom were treatment-refractory) were tested negative for antibodies against AChR by radioimmunoassay (RIA)], MuSK, LRP4 and AChR by cell binding assay, 5 (one of whom were treatment-refractory) against AChR (RIA) and MuSK and 1 against AChR (RIA) only

† Statistically significant

major comorbidities, time to immunosuppressive treatment initiation, thymectomy status or thymus histology. However, patients in the treatment-refractory group were younger (median age 33 vs $50.5, p=0.035$ ) and were more often classified as EOMG than LOMG. Multivariate regression analysis confirmed the statistically significant association of EOMG with a higher chance of developing treatmentrefractory MG ( $p=0.011$, OR 8.35, 95\% CI 1.64-42.64). Additionally, male sex was also associated with treatment failure in the multivariate analysis ( $p=0.011$, OR $6.05,95 \%$
CI 1.51-24.15), but not in the univariate analysis $(p=0.26)$. Antibody status and presence of thymoma in thymus histology were not significantly associated with treatment-refractory myasthenia gravis in the multivariate analyses.

Results of secondary outcome measures are shown in Table 2. Treatment-refractory patients had significantly higher maximum MGFA classes during their course of disease, $85.7 \%$ of these patients received escalation treatment with either rituximab or cyclophosphamide at some point and they required more rescue treatments with IVIG or PLEX/IA 
Table 2 Results of secondary outcome measures

Treatment-refractory MG $(n=14)$

Treatment-responsive MG

$p$ value* $(N=112)$

\begin{tabular}{ll}
\hline Myasthenic crisis & $2(14.3 \%)$ \\
Severe exacerbation & $9(64.3 \%)$ \\
Mortality & $2(14.3 \%)$ \\
MGFA at last FU & \\
Asymptomatic & $1(7.1 \%)$ \\
1 & $3(21.4 \%)$ \\
2 & $6(42.9 \%)$ \\
3 & $4(28.6 \%)$ \\
4 & 0 \\
5 & 0
\end{tabular}

$8(7.1 \%)$
$30(26.8 \%)$
$10(8.9 \%)$
$77(68.8 \%)$
$7(6.3 \%)$
$24(21.4 \%)$
$4(3.6 \%)$
0
0

MGFA-PIS at last FU**

\section{CSR}

$12(10.7 \%)$

PR

MM-0

0

MM-1

MM-2

$6(5.4 \%)$

MM-3

$1(7.1 \%)$

90.5 months (IQR 104)

Median time onset to FU

Median number of rescue treatments per patient with IVIG, PLEX or IA

Median number of different IS treatments

3 (IQR 6)

Escalation IS treatment***

3 (IQR 1)

$12(85.7 \%)$

$8(57.1 \%)$

Side effects of IS treatment

Treatment at last FU

$\begin{array}{ll}\text { Pyridostigmine } & 12(85.7 \%) \\ \text { IS treatment } & 14(100 \%) \\ \text { Maintenance IVIG/PLEX/IA } & 5(35.7 \%)\end{array}$

$38(33.9 \%)$

70 months (IQR 67) $\quad 0.047$

0.5 (IQR 1) $\quad \mathbf{0 . 0 0 2}$

$\begin{array}{ll}2(\mathrm{IQR} 1) & <\mathbf{0 . 0 0 0}^{\ddagger} \\ 6(5.4 \%) & <\mathbf{0 . 0 0 0}^{\ddagger} \\ 42(37.5 \%) & 0.23 \\ & \\ 73(65.2 \%) & 0.12 \\ 84(75 \%) & 0.034 \\ 3(2.7 \%) & <\mathbf{0 . 0 0 0}\end{array}$

Results of secondary outcome measures. Significance level after correction for multiple comparisons (Bonferroni correction) is $P \leq 0.004$

CSR denotes complete stable remission, $F U$ follow-up, IA immunoadsorption, IS immunosuppressive, IVIG intravenous immunoglobulins, $M G$ myasthenia gravis, MGFA Myasthenia Gravis Foundation of America, MM minimal manifestation, NA not applicable, PIS postintervention status, PLEX plasma exchange therapy and PR pharmacologic remission

${ }^{*} p$ values were obtained with the Mann-Whitney $U$ or Student's $t$ test (for continuous variables) and the Chi-squared test (for categorical variables) as appropriate

**16 patients in the treatment-responsive group did not meet the time criterion (duration of at least 1 year) of MGFA-PIS definitions

***Escalation IS treatment was defined as treatment with rituximab or cyclophosphamide

${ }^{\ddagger}$ Statistically significant

(median 3 times vs. 0.5, $p=0.002$ ). At the last follow-up after a median of 70 months for the treatment-responsive group and 90.5 months for treatment-resistant group, patients who had met the criteria for treatment-refractory myasthenia gravis at any point during the course of disease still had higher MGFA classes than treatment-responsive patients. Furthermore, all treatment-refractory patients required ongoing immunosuppressive treatment and $35.7 \%$ continued to receive additional maintenance treatment with IVIG or PLEX/IA. While we found no statistically significant differences in occurrence of myasthenic crisis or mortality, treatment-refractory patients had significantly higher numbers of severe myasthenic exacerbations. Treatment-related side effects occurred in $37.5 \%$ of treatment-responsive patients compared to $57.1 \%$ in treatment-refractory patients, but the difference was not statistically significant.

\section{Discussion}

In this study, we retrospectively investigated the frequency and clinical features of patients with treatment-refractory generalized myasthenia gravis. We found that $11.1 \%$ of our study population met the respective criteria indicating 
persistent myasthenic symptoms despite adequate treatment with at least two concurrent immunosuppressive drugs, usually steroids plus a steroid-sparing agent for at least the last year before assessing resistance. The other main finding of the study was that patients who became treatment-refractory during their course of disease, still had a worse outcome at last follow-up despite more aggressive ensuing treatment.

In comparison with previous studies, the percentage of treatment-refractory myasthenia gravis in our cohort is within the reported range of 9.2-14.8\% [12-14]. In contrast to these studies, however, we excluded patients with pure ocular myasthenia gravis, thus our findings are applicable only to patients with generalized symptoms developing within the first year of the disease. As detailed above, we used a strict definition of treatment-refractory myasthenia gravis based on the duration of treatment and symptoms, which partly differs from previously suggested criteria [9, 12-15]. Most importantly, we limited our definition to moderate to severe patients (i.e., a MGFA class of III or more). While we acknowledge that patients with persistent milder symptoms might also be affected to a relevant degree in their abilities of daily living and that these patients are potentially better captured by more lenient proposed definitions of refractory myasthenia gravis [18], we believe that our criteria complement previous criteria because they specifically target refractory patients who are clinically severely affected despite aggressive immunosuppressive treatment.

It is noteworthy that we also excluded patients who did not tolerate an adequate immunosuppressive therapy in contrast to definitions suggested by other authors [9]. We believe that these patients represent a different subgroup and should therefore be investigated separately as treatment-intolerant rather than refractory.

Our results suggest that patients with an onset of the disease before the age of 50 years might have a higher chance of becoming treatment-refractory than older patients. This confirms the results of Suh et al. [13] and is indirectly supported by a previous study reporting better outcome in older patients [7] but in contrast to other studies [19]. Varying rates of immunosuppressive treatments in older patients at different neuromuscular centers might explain the conflicting results. In comparison to previous reports that investigated treatment-refractory patients $[13,14]$, our findings differ insofar as we could not find an association of thymomaassociated myasthenia gravis, presence of MuSK-antibody or female sex with treatment-refractory myasthenia gravis. However, given the small number of patients in the respective subgroups, these findings should be interpreted with caution. The question if male or female sex is a risk factor, particularly warrants further investigation given the discrepancy between the multivariate analysis in our study suggesting an association of male sex with treatment-refractory myasthenia gravis, which was not evident in the univariate analysis, and previous outcome studies reporting no influence of sex [7, 19]. Nonetheless, our finding that MuSKantibody myasthenia gravis is not associated with a higher risk for refractory myasthenia gravis most likely reflects recent changes in treatment practices, which favor early treatment with rituximab leading to improved outcomes in this subgroup [20].

The frequency of myasthenic crises in patients without refractory disease was similar to previously reported cohorts [11] with $7.1 \%$ of patients experiencing at least one episode. Likewise, the rate of myasthenic crises in the refractory myasthenia gravis group (14.3\%) was only slightly lower than the reported number for treatment-refractory patients in the REGAIN study (18\%, cohort limited to AChR antibody positive myasthenia gravis) and the study by EngelNitz et al. (21.3\%) [11, 15]. Furthermore, the high number of severe exacerbations (64.3\%) in our cohort of treatmentrefractory patients was comparable to the REGAIN population, where $78 \%$ of patients reported any exacerbation of myasthenia gravis before enrollment and to the $71.2 \%$ reported by Engel-Nitz and colleagues.

Concerning treatment, refractory patients expectedly received on average more immunosuppressive drugs and also more escalation therapies, but the time to immunosuppressive treatment initiation did not differ between the groups. Since previous studies showed an association of a shorter time to diagnosis with better remission, additional studies are needed to investigate if faster and more aggressive treatment approaches are beneficial in certain myasthenia gravis subgroups. The frequent exacerbations in treatment-refractory patients were also accompanied by a substantially higher number of rescue treatments. However, despite the higher number of both standard and escalation immunosuppressive treatments, only one patient in the treatment-refractory group was clinically asymptomatic at last follow-up, all patients required ongoing immunosuppressive treatments and about a third continued to be dependent on additional maintenance treatment with either IVIG or PLEX/ IA, further emphasizing the enormous impact on health care systems and the individual patient.

This study has some inherent limitations. First, due to the retrospective design, patient data regarding clinical deterioration could have been missed, but to minimize this effect we only included patients with sufficient clinical information available. Second, our cohort represents a myasthenia gravis population at a specialized tertiary department, therefore we cannot exclude a selection bias towards more severely affected patients. Furthermore, time to last follow-up was slightly shorter in the treatment-responsive group, thus it cannot be excluded that some patients of this group would have become treatment-refractory after the last follow-up. However, most patients met the criteria considerably earlier than at the 
Table 3 Characteristics of treatment-refractory patients

\begin{tabular}{|c|c|c|c|c|c|c|c|c|}
\hline & Sex & Age at onset & Antibody & Thymectomy & Histology & $\begin{array}{l}\text { Time to } \\
\text { refractory MG } \\
(\text { mos.) }\end{array}$ & IS treatment at refractory timepoint & $\begin{array}{l}\text { MGFA } \\
\text { class at last } \\
\text { FU }\end{array}$ \\
\hline 1 & $\mathrm{~m}$ & 73 & $\mathrm{AChR}$ & No & NA & 60 & CS 5 mg, MMF $1000 \mathrm{mg}, \mathrm{CP}(1 \mathrm{~g} 4 \mathrm{w})$ & IIIa \\
\hline 2 & $\mathrm{~m}$ & 59 & $\mathrm{AChR}$ & No & NA & 56 & CS 75 mg, CP (1 g q4w), IA/PLEX & IIIb \\
\hline 3 & $\mathrm{~m}$ & 41 & MuSK & No & NA & 24 & CS $18.8 \mathrm{mg}$, TC $6 \mathrm{mg}$, IA/PLEX & IIb \\
\hline 4 & $\mathrm{~m}$ & 54 & $\mathrm{AChR}$ & Yes & Thymoma & 24 & CS $12.5 \mathrm{mg}$, AZA $150 \mathrm{mg}$, IA/PLEX & 0 (MM3) \\
\hline 5 & $\mathrm{f}$ & 22 & $\mathrm{SN}$ & Yes & Hyperplasia & 197 & CS $10 \mathrm{mg}, \mathrm{RTX}\left(375 \mathrm{mg} / \mathrm{m}^{2} \mathrm{BSA}\right)$ & IIa \\
\hline 6 & $\mathrm{f}$ & 35 & $\mathrm{AChR}$ & Yes & Thymoma & 74 & CS 25 mg, MMF $2000 \mathrm{mg}$ & IIa \\
\hline 7 & $\mathrm{f}$ & 27 & $\mathrm{AChR}$ & Yes & Normal & 24 & CS $8.75 \mathrm{mg}$, AZA $150 \mathrm{mg}$, IA/PLEX & IIb \\
\hline 8 & $\mathrm{~m}$ & 13 & $\mathrm{AChR}$ & Yes & Hyperplasia & 158 & MMF 150, RTX (375 mg/m² BSA), IA/PLEX & I \\
\hline 9 & $\mathrm{~m}$ & 49 & $\mathrm{AChR}$ & No & NA & 52 & CS 25 mg, AZA 200 mg, IVIG & $\mathrm{I}$ \\
\hline 10 & $\mathrm{~m}$ & 28 & $\mathrm{SN}$ & Yes & Hyperplasia & 49 & CS 25 mg, MMF 2000 mg, IVIG & I \\
\hline 11 & $\mathrm{f}$ & 27 & $\mathrm{AChR}$ & Yes & Normal & 24 & CS $25 \mathrm{mg}$, AZA $100 \mathrm{mg}$, IVIG & IIb \\
\hline 12 & $\mathrm{~m}$ & 48 & AChR & Yes & Thymoma & 25 & $\begin{array}{l}\text { CS } 17.5 \mathrm{mg} \text {, MMF } 2000 \mathrm{mg} \text {, RTX }\left(375 \mathrm{mg} / \mathrm{m}^{2}\right. \\
\text { BSA), IVIG }\end{array}$ & IIa \\
\hline 13 & $\mathrm{f}$ & 31 & $\mathrm{SN}$ & Yes & Normal & 25 & $\begin{array}{l}\text { CS } 20 \mathrm{mg} \text {, AZA } 100 \mathrm{mg} \text {, RTX }\left(375 \mathrm{mg} / \mathrm{m}^{2}\right. \\
\text { BSA); IA/PLEX }\end{array}$ & IIIa \\
\hline 14 & $\mathrm{f}$ & 20 & $\mathrm{AChR}$ & Yes & Hyperplasia & 40 & CS $12.5 \mathrm{mg}$, AZA $100 \mathrm{mg}$ & IIIb \\
\hline
\end{tabular}

Characteristics of treatment-refractory patients. Treatment is shown for the timepoint patients met the definition of refractory MG

$A C h R$ denotes acetylcholine receptor, $A Z A$ azathioprine, $B S A$ body surface area, $C P$ Cyclophosphamide, $C S$ corticosteroids, $F U$ follow-up, $I A / P L E X$ immunoadsorption or plasma exchange therapy (maintenance treatment), IS immunosuppressive, IVIG intravenous immunoglobulins (maintenance treatment), $M G$ myasthenia gravis, $M G F A$ Myasthenia Gravis Foundation of America, $M M$ minimal manifestation, $M M F$ mycophenolate mofetil, $M u S K$ muscle-specific receptor tyrosine kinase, $N A$ not applicable, $S N$ seronegative, $T C$ tacrolimus

last follow-up, arguing against a relevant bias (Table 3). Finally, the definition of treatment-refractory myasthenia gravis was clinically based on retrospectively rated MGFA classes, which partly depends on subjective assessment of clinical symptoms. Therefore, future studies are necessary to define treatment-refractory myasthenia gravis based on quantitative scores (i.e., myasthenia gravis composite score or the quantitative myasthenia gravis score [17]).

Summarizing our data, we found that despite a growing number of available treatments for myasthenia gravis and improved general care, about one tenth of patients still become treatment-refractory during their course of disease with considerable implications both for patients as well as health care providers. Future studies are necessary to find potential early biomarkers for this patient group given that currently no clinical feature has a high sensitivity or specificity in predicting treatment response.

Acknowledgments Open access funding provided by Medical University of Vienna. This study received no specific grant from any funding agency.

\section{Compliance with ethical standards}

Conflicts of interest No relevant conflicts of interest to disclose related to the article.
Ethical standard statement The study was approved by the local Ethics Committee of the Medical University of Vienna.

Open Access This article is licensed under a Creative Commons Attribution 4.0 International License, which permits use, sharing, adaptation, distribution and reproduction in any medium or format, as long as you give appropriate credit to the original author(s) and the source, provide a link to the Creative Commons licence, and indicate if changes were made. The images or other third party material in this article are included in the article's Creative Commons licence, unless indicated otherwise in a credit line to the material. If material is not included in the article's Creative Commons licence and your intended use is not permitted by statutory regulation or exceeds the permitted use, you will need to obtain permission directly from the copyright holder. To view a copy of this licence, visit http://creativecommons.org/licenses/by/4.0/.

\section{References}

1. Cetin H, Fülöp G, Zach H et al (2012) Epidemiology of myasthenia gravis in Austria: rising prevalence in an ageing society. Wien Klin Wochenschr 124:763-768. https://doi.org/10.1007/ s00508-012-0258-2

2. Gilhus NE, Verschuuren JJ (2015) Myasthenia gravis: subgroup classification and therapeutic strategies. Lancet Neurol 14:10231036. https://doi.org/10.1016/S1474-4422(15)00145-3

3. Marx A, Pfister F, Schalke B et al (2013) The different roles of the thymus in the pathogenesis of the various myasthenia gravis subtypes. Autoimmun Rev 12:875-884. https://doi.org/10.1016/j. autrev.2013.03.007 
4. Gilhus NE (2016) Myasthenia gravis. N Engl J Med 375:25702581. https://doi.org/10.1056/NEJMra1602678

5. Oosterhuis HJ (1989) The natural course of myasthenia gravis: a long term follow up study. J Neurol Neurosurg Psychiatry 52:1121-1127. https://doi.org/10.1136/jnnp.52.10.1121

6. Grob D, Brunner N, Namba T, Pagala M (2008) Lifetime course of myasthenia gravis. Muscle Nerve 37:141-149. https://doi. org/10.1002/mus.20950

7. Andersen JB, Gilhus NE, Sanders DB (2016) Factors affecting outcome in myasthenia gravis. Muscle Nerve 54:1041-1049. https ://doi.org/10.1002/mus.25205

8. Silvestri NJ, Wolfe GI (2014) Treatment-refractory myasthenia gravis. J Clin Neuromuscul Dis 15:167-178. https://doi. org/10.1097/CND.0000000000000034

9. Mantegazza R, Antozzi C (2018) When myasthenia gravis is deemed refractory: clinical signposts and treatment strategies. Ther Adv Neurol Disord 11:1756285617749134. https://doi. org/10.1177/1756285617749134

10. Schneider-Gold C, Hagenacker T, Melzer N, Ruck T (2019) Understanding the burden of refractory myasthenia gravis. Ther Adv Neurol Disord 12:1756286419832242. https://doi. org/10.1177/1756286419832242

11. Engel-Nitz NM, Boscoe A, Wolbeck R et al (2018) Burden of illness in patients with treatment refractory myasthenia gravis. Muscle Nerve. https://doi.org/10.1002/mus.26114

12. Xin H, Harris LA, Aban IB, Cutter G (2019) Examining the Impact of refractory myasthenia gravis on healthcare resource utilization in the united states: analysis of a Myasthenia Gravis Foundation of America Patient Registry Sample. J Clin Neurol 15:376-385. https://doi.org/10.3988/jen.2019.15.3.376

13. Suh J, Goldstein JM, Nowak RJ (2013) Clinical characteristics of refractory myasthenia gravis patients. Yale J Biol Med $86: 255-260$
14. Sudulagunta SR, Sepehrar M, Sodalagunta MB et al (2016) Refractory myasthenia gravis - clinical profile, comorbidities and response to rituximab. Ger Med Sci 14:12. https://doi. org/10.3205/000239

15. Howard JF, Utsugisawa K, Benatar M et al (2017) Safety and efficacy of eculizumab in anti-acetylcholine receptor antibodypositive refractory generalised myasthenia gravis (REGAIN): a phase 3 , randomised, double-blind, placebo-controlled, multicentre study. Lancet Neurol 16:976-986. https://doi.org/10.1016/ S1474-4422(17)30369-1

16. Muppidi S, Utsugisawa K, Benatar M et al (2019) Long-term safety and efficacy of eculizumab in generalized myasthenia gravis. Muscle Nerve 60:14-24. https://doi.org/10.1002/ mus. 26447

17. Benatar M, Sanders DB, Burns TM et al (2012) Recommendations for myasthenia gravis clinical trials. Muscle Nerve 45:909-917. https://doi.org/10.1002/mus.23330

18. Sanders DB, Wolfe GI, Benatar M et al (2016) International consensus guidance for management of myasthenia gravis: executive summary. Neurology 87:419-425. https://doi.org/10.1212/ WNL.0000000000002790

19. Mao Z-F, Mo X-A, Qin C et al (2010) Course and prognosis of myasthenia gravis: a systematic review. Eur J Neurol 17:913-921. https://doi.org/10.1111/j.1468-1331.2010.03017.x

20. Hehir MK, Hobson-Webb LD, Benatar M et al (2017) Rituximab as treatment for anti-MuSK myasthenia gravis: multicenter blinded prospective review. Neurology 89:1069-1077. https://doi. org/10.1212/WNL.0000000000004341 